Short research communication

\title{
Multimycotoxin analysis of South African Aspergillus clavatus isolates
}

Authors:

C. J. Botha ${ }^{1}$

M. Truter $^{2}$

M. Sulyok ${ }^{3}$

\section{Affiliations:}

${ }^{1}$ Department of Paraclinical Sciences, Faculty of Veterinary Science, University of Pretoria, Private bag X04, Onderstepoort, 0110, South Africa

${ }^{2}$ Biosystematics Division, Agricultural Research Council-Vegetable and Ornamental Plants, Private Bag X293, Pretoria, 0001, South Africa

${ }^{3}$ Centre for Analytical Chemistry, Department of Agrobiotechnology (IFA-Tulln), University of Natural Resources and Life Sciences, Konrad Lorenzstr. 20, A-3430, Tulln, Austria

CJ Botha

christo.botha@up.ac.za

Tel.: +27 125298023; fax +27 125298304 .

ORCID CJ Botha 0000000315359270 


\section{Abstract}

Aspergillus clavatus poisoning is a neuromycotoxicosis of ruminants that occurs sporadically across the world after ingestion of infected feedstuffs. Although various toxic metabolites are synthesized by the fungus it is not clear which specific or group of mycotoxins induces the syndrome. A. clavatus isolates were deposited in the culture collection of the Biosystematics Division, Plant Protection Research Institute, Agricultural Research Council during incidences of livestock poisoning $(1988-2016)$. Six isolates were still viable and these plus three other South African isolates that were also previously deposited in the collection were positively identified as $A$. clavatus based on morphology and B-tubulin sequence data. The cultures were screened for multiple mycotoxins using a liquid chromatography-tandem mass spectrometric (LC-MS/MS) method. Twelve A. clavatus metabolites were detected. The concentrations of the tremorgenic mycotoxins (i.e. tryptoquivaline $A$ and its related metabolites deoxytryptoquivaline $\mathrm{A}$ and deoxynortryptoquivaline) were higher than patulin and cytochalasin E. Livestock owners should not feed $A$. clavatus-infected material to ruminants as all the South African A. clavatus isolates synthesized the same compounds when cultured under similar conditions.

Keywords Aspergillus clavatus, cytochalasin E, patulin, tremorgenic, tryptoquivaline 


\section{Introduction}

Aspergillus clavatus poisoning in ruminants is a neuromycotoxicosis resulting in irreversible neuronal damage and is associated with a high mortality rate (Kellerman et al. 2005). In South Africa it occurs sporadically in cattle and follows the ingestion of mould-infected sorghum beer residue ('maroek') or infected sprouting grain (Kellerman et al. 2005). Periodic outbreaks have also been reported from across the world (McKenzie et al. 2004; Riet-Correa et al. 2013; Sabater-Vilar et al. 2004; Schlosberg et al. 1991).

Aspergillus clavatus synthesizes various metabolites which are potentially toxic such as patulin (Lopez-Diaz and Flannigan 1997; Sabater-Vilar et al. 2004) and cytochalasin E (Büchi et al. 1973; Lopez-Diaz and Flannigan 1997) as well as the tremorgenic metabolites such as tryptoquivalone (Clardy et al. 1975) and tryptoquivaline and its related metabolites (Büchi et al. 1977; Clardy et al. 1975). However, none of these has been administered to ruminants to ascertain toxicity and to apportion responsibility as the cause of the syndrome (McKenzie et al. 2004). Thus, there is still uncertainty as to which specific mycotoxin or group of mycotoxins induces this neuromycotoxicosis in livestock (Botha et al. 2014).

Some of the A. clavatus isolates deposited in the National Collection of Fungi (NCF), Biosystematics Division, Plant Protection Research Institute, Agricultural Research Council (ARC-PPRI), Pretoria, South Africa have previously been associated with intoxication of livestock. If the isolates that have previously been associated with livestock intoxication presented consistently higher concentrations compared to the other isolates in the collection, it could contribute to clarify which specific or multiple mycotoxins are involved in this particular intoxication. The objective of this study was to submit cultures of all viable South African A. clavatus isolates available in the collection for multimycotoxin analysis. 


\section{Materials and methods}

\section{Culturing of isolates}

Nine viable isolates that had been deposited from 1988 to 2016 in the NCF were cultured. Isolates were originally isolated mainly from plant material collected from Free State, Gauteng, Mpumalanga, North West and Western Cape Provinces (Table 1). Aspergillus clavatus isolates were plated to Malt Extract Agar (MEA) (Klich 2002) and incubated in the dark at $25 \stackrel{\circ}{\mathrm{C}}$ for $14 \mathrm{~d}$.

\section{Identification of isolates}

The isolates were grown for $7 \mathrm{~d}$ as 3-point inoculations on Czapek yeast extract agar (CYA), Czapek yeast extract agar with $20 \%$ sucrose (CY20S) and MEA at $25^{\circ} \mathrm{C}$ in the dark (Klich 2002; Samson et al. 2014). Microscopic characteristics were observed from MEA on day seven. Structures were mounted in $70 \%$ lactic acid and microscopic characteristics observed at 100x and 400x magnification under differential interference contrast (DIC) using a Zeiss Axio Imager compound microscope fitted with an AxioCam MRc camera.

The isolates were grown on MEA for 7 days at $25^{\circ} \mathrm{C}$ in the dark. DNA were isolated using the DNeasy plant mini-extraction kit (Qiagen, Valencia, CA, USA) by following the manufacturer's protocol after the mycelium was placed in Eppendorf tubes and manually ground with ca. $10 \mu \mathrm{g}$ sterile, chemically treated sand. Amplification of part of the $\beta$-tubulin gene was performed using the primers Bt2a and Bt2b as described by Glass and Donaldson (1995). The resulting PCR amplicons were submitted to Inqaba Biotech (Pretoria, South Africa) for sequencing. The $\beta$-tubulin sequences were deposited at the GenBank nucleotide sequence database under accession numbers MF319755-MF319763.

All six species in the Aspergillus section Clavati based on Varga et al. (2007) were included in the data set and aligned with MAFFT v.7 (http://mafft.cbrc.jp/alignment/server/ 
index.html). Gaps were treated as missing data in the subsequent analysis. Phylogenetic analysis were based on maximum parsimony (MP) using PAUP 4.0* (Phylogenetic Analysis Using Parsimony* and Other Methods version 4, Swofford, 2002), using the heuristic search option with 100 random taxa additions and tree bisection and reconstruction (TBR) as the branch-swapping algorithm. Branches of zero length were collapsed and all multiple, equally parsimonious trees were saved. Bootstrap analyses were performed to determine branching point confidence intervals (1000 replicates) for the most parsimonious trees generated for the respective data set. An Aspergillus fischeri isolate was used as the outgroup in the analysis (Varga et al. 2007).

\section{Multimycotoxin analysis}

Plugs of MEA (0.4 - $0.6 \mathrm{~g})$ containing fungal growth were extracted using acetonitrile/water/acetic acid 79/20/1 (v/v/v). The diluted extracts were screened for target fungal metabolites with a QTrap 5500 LC-MS/MS System (Applied Biosystems, Foster City, CA, USA) equipped with a Turbolon Spray electrospray ionization (ESI) source and a 1290 Series HPLC System (Agilent, Waldbronn, Germany). Chromatographic separation was performed at $25{ }^{\circ} \mathrm{C}$ on a Gemini $\mathrm{C}_{18}$-column, $150 \times 4.6 \mathrm{~mm}$ i.d., $5 \mu \mathrm{m}$ particle size, equipped with a $\mathrm{C}_{18} 4 \times 3 \mathrm{~mm}$ i.d. security guard cartridge (all from Phenomenex, Torrance, CA, USA).

The chromatographic method as well as chromatographic and mass spectrometric parameters are described in (Malachova et al. 2014), but the method has in the meantime been expanded to cover more than 650 metabolites (manuscript in preparation). Besides detecting tryptoquivaline $\mathrm{A}$ and its metabolites, the analytical method included other tremorgenic compounds, i.e. ergot alkaloids, clavinet alkaloids, lolitrem B and N, paxilline and desoxypaxilline, paspaline, paspalinine, paspalitrem A and B and penitrem A, as well.

Electrospray ionization-tandem mass spectrometry (ESI-MS/MS) was performed in the time-scheduled multiple reaction monitoring (MRM) mode both in positive and negative 
polarities in two separate chromatographic runs per sample by scanning two fragmentation reactions per analyte. The MRM detection window of each analyte was set to its expected retention time $\pm 27 \mathrm{sec}$ and $\pm 48 \mathrm{sec}$ in the positive and the negative mode, respectively. Confirmation of positive analyte identification was obtained by the acquisition of two MRMs per analyte (with the exception of moniliformin and 3-nitropropionic acid that exhibited only one fragment ion), which yielded 4.0 identification points according to commission decision 2002/657/EC. In addition, the liquid chromatography (LC) retention time and the intensity ratio of the two MRM transition agreed with the related values of an authentic standard within $0.1 \mathrm{~min}$ and $30 \%$ rel., respectively. The accuracy of the method is verified on a continuous basis by regular participation in proficiency testing schemes (Malachova et al. 2014, 2015). Quantification was performed via external calibration using serial dilutions of a multi-analyte stock solution.

\section{Statistical analysis}

Data were analysed using the statistical program GenStat (Payne 2014). The data were logtransformed and a $t$-test performed. A Mann Whitney test was also performed on the untransformed data. Significance was set at $p<0.05$.

\section{Results}

\section{Identification of isolates}

Aspergillus clavatus isolates had colony diameters of $42-50 \mathrm{~mm}$ on CYA, $35-48 \mathrm{~mm}$ on MEA and $38-50 \mathrm{~mm}$ on CY20S at $25^{\circ} \mathrm{C}$. Colony diameters at $37^{\circ} \mathrm{C}$ were $13-26 \mathrm{~mm}$ on CYA. Mycelium white and usually inconspicuous, whereas conidia were dull green to greyish turquoise. Stipes were 500-1500 x 15-30 $\mu \mathrm{m}$, smooth-walled, colourless, and expanding gradually into clavate vesicles, 35-75 $\mu \mathrm{m}$ wide (Fig. 1a). The club-shaped vesicle of $A$. 
clavatus (Fig. 1a) can clearly be distinguish from the spherical vesicle of $A$. niger (Fig. 1b). Conidial zone extended from 50-180 $\mu \mathrm{m}$ down from the top of the vesicles; heads were uniseriate with phialides 7-9 x 3.5 $\mu \mathrm{m}$. Conidia were smooth-walled, ellipsoidal, 4-6 x 3-4 $\mu \mathrm{m}$, and dull turquoise in mass.
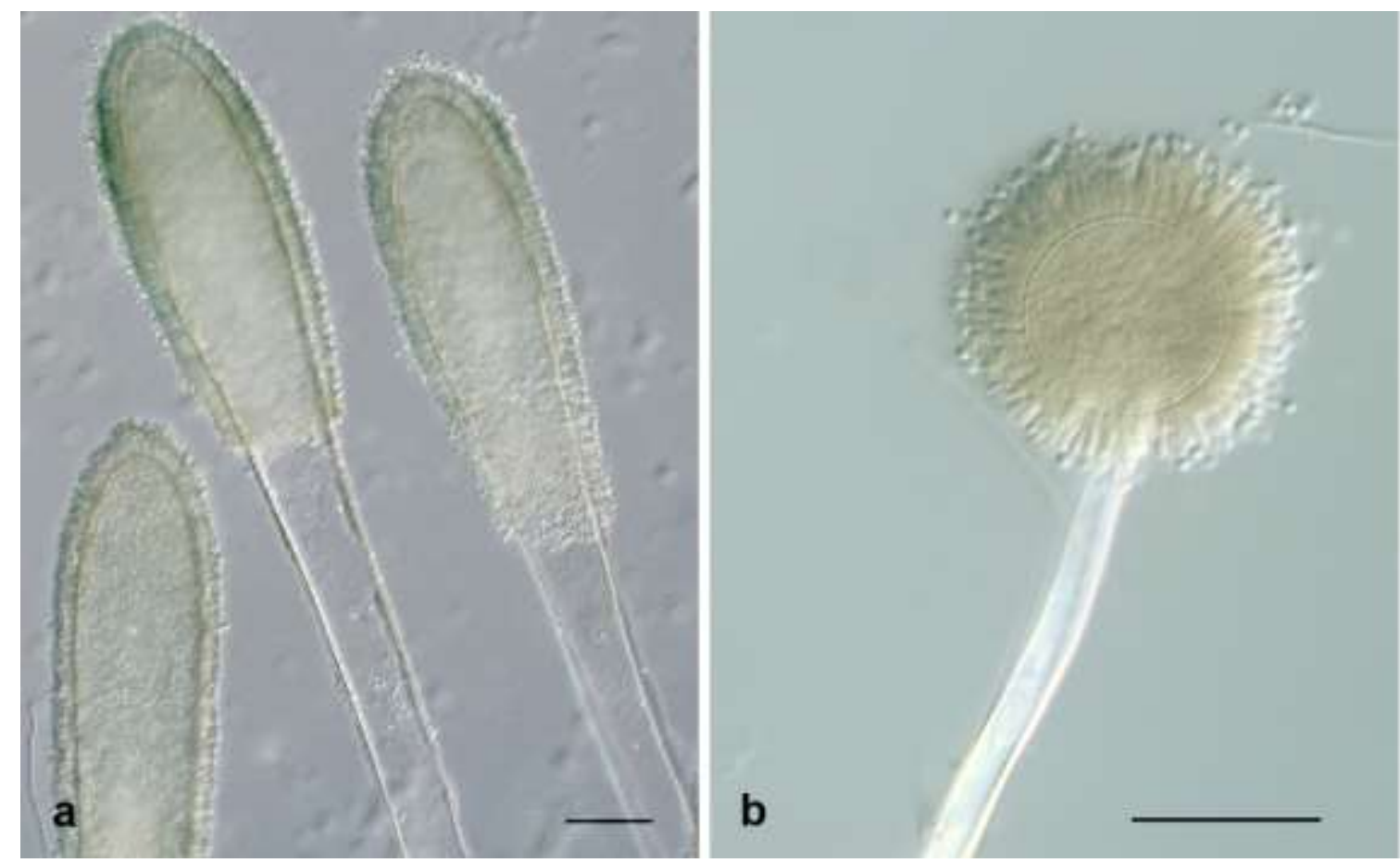

Fig. 1a The club-shaped conidiophores of Aspergillus clavatus (PPRI 21896) and (b) the spherical conidiophore of Aspergillus niger (b) mounted in lactic acid, observed under differential interference contrast (DIC) using a Zeiss Axio Imager compound microscope fitted with an AxioCam MRc camera, scale bar a $50 \mu \mathrm{m}$

Multi-sequence alignment by inserting gaps resulted in a total of 467 characters. All parsimony-uninformative and constant characters were excluded, resulting in 79 parsimonyinformative characters. All nine PPRI isolates grouped within the A. clavatus clade with a $100 \%$ bootstrap support value, and are clearly separate from the other species in the section Clavati (Fig. 2). 


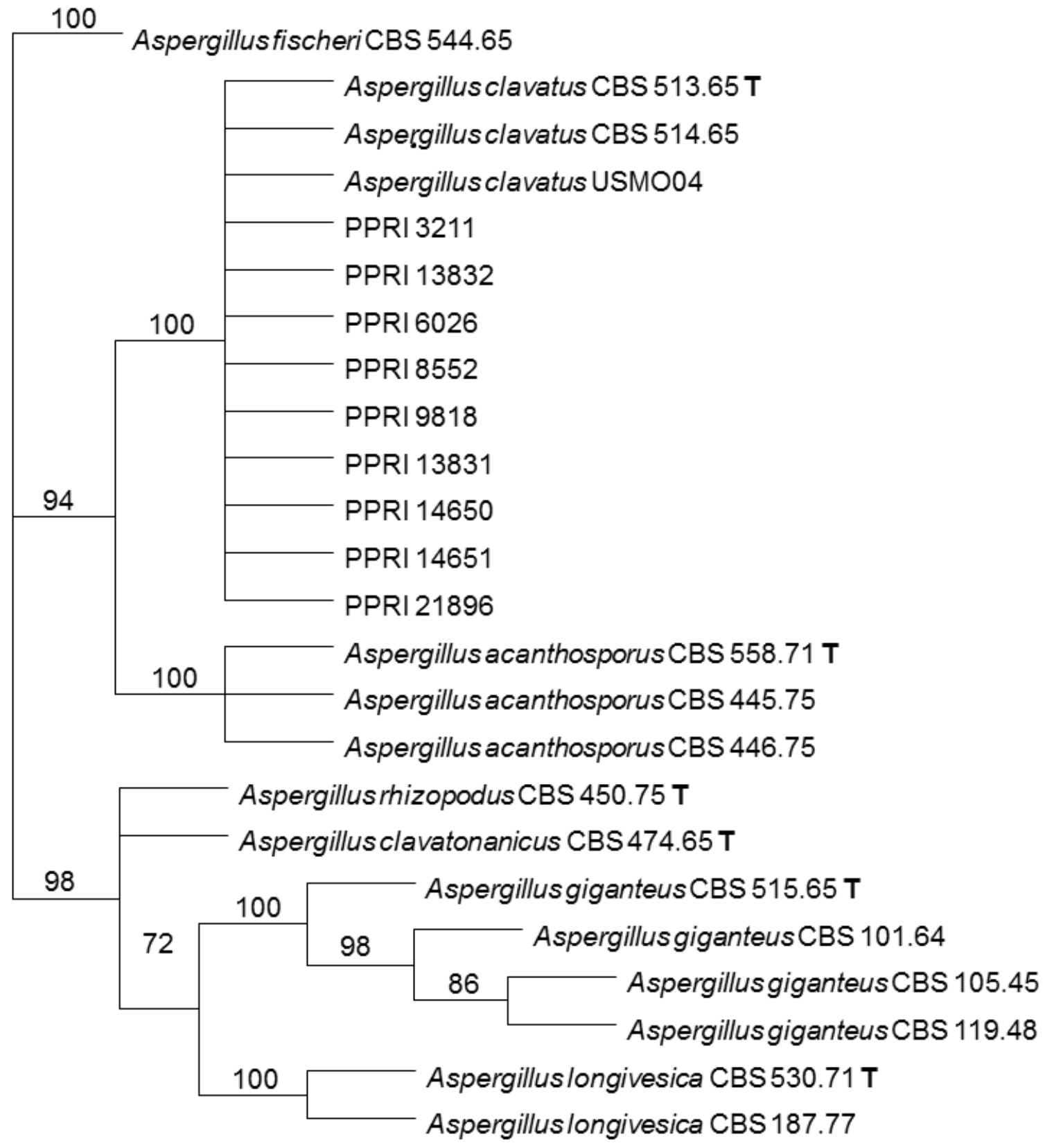

Fig. 2 One of the most parsimonious trees based on the partial B-tubulin sequences of isolates within Aspergillus section Clavati. The maximum parsimony bootstrap support values from 1000 replicates are given at the nodes, with values $\geq 70 \%$ shown. Ex-type strains are indicated indicated with $\mathrm{T}$ after the isolate number. The tree was rooted to Aspergillus fischeri (CBS 544.65) 
<smiles>O=c1cc2occcc-2o1</smiles>

Patulin

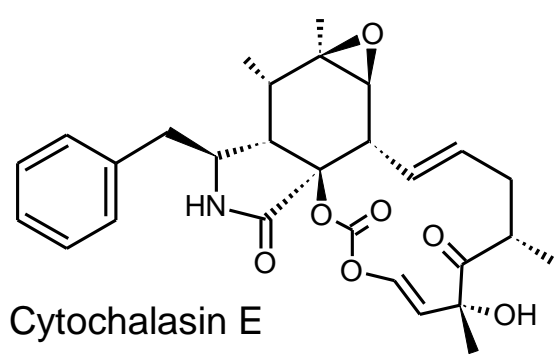<smiles>[Y7]N1[C@H]2N(C(=O)C1(C)C)c1ccccc1[C@@]21C[C@@H](n2c([C@H](OC(C)=O)C(C)C)nc3ccccc3c2=O)C(=O)O1</smiles><smiles>CCCCCCC(O)CCCCCC/C=C/[C@@H](O)[C@H](O)[C@H](O)[C@H]1NC(=O)OC1=O</smiles>

$\mathrm{R}^{1}=\mathrm{OH}, \mathrm{R}^{2}=\mathrm{CH}_{3}$ Tryptoquivaline $\mathrm{A}$ $\mathrm{R}^{1}=\mathrm{OH}, \mathrm{R}^{2}=\mathrm{H}$ Nortryptoquivaline $\mathrm{A}$ $R^{1}=R^{2}=H$ Deoxynortryptoquivaline $A$

Sphingofungin D<smiles>CCCCCCCOc1ccccc1C(=O)NC(=C(C)C)c1nc2ccccc2c(=O)n1CC1(O)c2ccccc2N2C(=O)C(C)(C)NC21</smiles><smiles>O=C1N[C@H](Cc2ccc(O)cc2)C(=O)N2CCC[C@H]12</smiles><smiles>CC(C)[C@@H]1NC(=O)[C@H]2CCCN2C1=O</smiles>

Cyclo(L-Pro-L-Tyr)

Cyclo(L-Pro-L-Val)<smiles>O=C1N[C@H](Cc2c[nH]c3ccccc23)C(=O)N2CCC[C@H]12</smiles>

Brevianamide $\mathrm{F}$<smiles>O=C1N[C@H](Cc2c[nH]c3ccccc23)C(=O)N[C@@H]1Cc1ccccc1</smiles>

Rugulusovine

Fig. 3 The chemical structures of the mycotoxins detected after screening of the $A$. clavatus isolates with liquid chromatography-tandem mass spectrometry (LC-MS/MS) 


\section{Multimycotoxin analysis}

Patulin concentration detected in all isolates ranged from approximately 3500 - $74000 \mathrm{ng}$ per g mycelium/agar and tryptoquivaline A from roughly $20500-107000$ ng/g. The concentrations of all the tremorgenic mycotoxins (i.e. tryptoquivaline $\mathrm{A}$ and its related metabolites - deoxytryptoquivaline A and deoxynortryptoquivaline) were relatively high. The metabolites identified and their respective concentrations are listed in Table 1 and the chemical structures are illustrated in Fig. 3. The commercial sources of the standards for the positively identified compounds were: Analyticon Discovery (Potsdam, Germany) for the tryptoquivaline derivatives and sphingofungin D; Romer Labs (Tulln, Austria) for patulin; Sigma (Vienna, Austria) for cytochalasin E and Bio Australis (Smithfield, Australia) for the cyclic dipeptides, whereas quinadoline A was obtained as a gift.

\section{Discussion}

Twelve A. clavatus metabolites, which included major Aspergillus mycotoxins (patulin, tryptoquivaline and cytochalasin E) as well as non-specific metabolites, were detected (Table 1). All the potential $A$. clavatus mycotoxins and other metabolites detected in isolates previously associated with livestock poisoning were also synthesized by the other South African isolates that were deposited in the culture collection. No significant differences $(p>$ $0.05)$ between the concentrations of mycotoxins in both groups were observed.

When the different fungal metabolite concentrations were compared (Table 1) the concentrations of the tremorgenic mycotoxins (i.e. tryptoquivaline $\mathrm{A}$ and metabolites) were higher than patulin. On the contrary, patulin has been suggested as a potential cause of this intoxication (Riet-Correa et al. 2013; Sabater-Vilar et al. 2004). The tremorgenic mycotoxin concentrations were also much higher than cytochalasin E, in spite of a report indicating that cytochalasin E is mainly synthesized at $25^{\circ} \mathrm{C}$ (Lopez-Diaz and Flannigan 1997), the incubation temperature used to culture the isolates in this investigation. Nevertheless, when 
Table 1 Aspergillus clavatus metabolites and estimated concentrations $(\mu \mathrm{g} / \mathrm{kg})$ of isolates $(\mathrm{n}=9)$ plated on Malt Extract Agar and incubated in the dark at 25 ${ }^{\circ} \mathrm{C}$ for $14 \mathrm{~d}$. The isolates were deposited in the National Collection of Fungi, Biosystematics Division, Plant Protection Research Institute, Agricultural Research Council (ARC-PPRI), Pretoria, South Africa. LOD = limit of detection

South African isolates associated with livestock intoxication

\begin{tabular}{|c|c|c|c|c|c|c|c|c|c|c|c|c|c|}
\hline $\begin{array}{l}\text { PPRI } \\
\text { isolate } \\
\text { no. }\end{array}$ & Province and host/substrate & Patulin & Cyto $E^{a}$ & Sphin $D^{b}$ & Tryp Ac & Deoxytryp Ad & $\begin{array}{l}\text { Deoxy- } \\
\text { Nortrype }\end{array}$ & Tryptoquialf $^{f}$ & Quin $A^{g}$ & Brev Fh & $\begin{array}{l}\text { cyclo(L-Pro- } \\
\text { L-Tyr) }\end{array}$ & $\begin{array}{l}\text { cyclo(L-Pro- } \\
\text { L-Val) }\end{array}$ & Ruguli \\
\hline 6026 & North West, unknown & 7400 & 2200 & 140 & 103200 & 70600 & 31800 & 6100 & 13300 & 310 & 4000 & 3700 & 90 \\
\hline $13831 j$ & Gauteng, barley sprouts & 19600 & 1400 & 110 & 52800 & 37800 & 20500 & 4900 & 10200 & 300 & 4100 & 3800 & 70 \\
\hline $13832^{j}$ & Gauteng, barley sprouts & 3400 & 2500 & 90 & 45700 & 29400 & 16600 & 3300 & 6300 & 300 & 4000 & 3600 & 70 \\
\hline 14650 & North West, feed pellets & 17900 & 3000 & 120 & 107200 & 62900 & 17700 & 8400 & 15800 & 310 & 3900 & 4000 & 70 \\
\hline 14651 & North West, feed pellets & 57600 & 3600 & 120 & 62700 & 41100 & 29100 & 9900 & 22000 & 280 & 4200 & 3900 & 70 \\
\hline 21896 & Western Cape, barley sprouts & 39500 & 2300 & 20 & 20500 & 8200 & 17100 & 2400 & 5100 & 140 & 2100 & 1700 & 40 \\
\hline Median & & 18750 & 2400 & 115 & 57750 & 39450 & 19100 & 5500 & 11750 & 300 & 4000 & 3750 & 70 \\
\hline \multicolumn{14}{|c|}{ Other South African isolates } \\
\hline $\begin{array}{l}\text { PPRI } \\
\text { isolate } \\
\text { no. }\end{array}$ & Province and host/substrate & Patulin & Cyto $E^{a}$ & Sphin $D^{b}$ & Tryp Ac & Deoxytryp Ad & $\begin{array}{l}\text { Deoxy- } \\
\text { Nortrype }\end{array}$ & Tryptoquialf $^{f}$ & Quin $A^{g}$ & Brev Fh & $\begin{array}{l}\text { cyclo(L-Pro- } \\
\text { L-Tyr) }\end{array}$ & $\begin{array}{l}\text { cyclo(L-Pro- } \\
\text { L-Val) }\end{array}$ & Ruguli \\
\hline 3211 & Free State, grass roots & 5000 & 1500 & 50 & 42500 & 29000 & 26000 & 5600 & 12400 & 270 & 4100 & 4100 & 70 \\
\hline 8552 & Mpumalanga, sunflower seeds & 73800 & 2700 & 60 & 67100 & 43600 & 14100 & 5200 & 11000 & 260 & 3900 & 3900 & 60 \\
\hline 9818 & Free Sate, soil & 15200 & 2500 & 180 & 72000 & 39600 & 59700 & 7700 & 17900 & 287 & 4000 & 3700 & 70 \\
\hline Median & & 15200 & 2500 & 60 & 67100 & 39600 & 26000 & 5600 & 12400 & 270 & 4000 & 3900 & 70 \\
\hline
\end{tabular}


aCytochalasin E (LOD $=0.5 \mathrm{ng} / \mathrm{g})$

${ }^{b}$ Sphingofungin $\mathrm{D}(\mathrm{LOD}=0.64 \mathrm{ng} / \mathrm{g})$

cTryptoquivaline $\mathrm{A}(\mathrm{LOD}=0.8 \mathrm{ng} / \mathrm{g})$

Deoxytryptoquivaline A (LOD = $2 \mathrm{ng} / \mathrm{g}$ )

eDeoxynortryptoquivaline (LOD $=2 \mathrm{ng} / \mathrm{g}$ )

Tryptoquialanine Derivative (LOD $=0.24 \mathrm{ng} / \mathrm{g}$ )

'Quinadoline A (LOD = $2 \mathrm{ng} / \mathrm{g}$ )

hBrevianamide $\mathrm{F}(\mathrm{LOD}=0.5 \mathrm{ng} / \mathrm{g})$

cyclo(L-Pro-L-Tyr) (LOD $=0.8 \mathrm{ng} / \mathrm{g})$

cyclo(L-Pro-L-Val) $(L O D=0.64 \mathrm{ng} / \mathrm{g})$

Rugulusovine (LOD $=0.3 \mathrm{ng} / \mathrm{g}$ )

i Source: Botha et al. 2014 
the acute toxicities of these mycotoxins for rodents are compared it appears that patulin and cytochalasin $\mathrm{E}$ are more toxic than tryptoquivaline. Following intraperitoneally administration to weanling rats, $500 \mathrm{mg} / \mathrm{kg}$ tryptoquivaline metabolites caused mortality within $8 \mathrm{~d}$ whereas only 2.6 mg cytochalasin E per kg caused mortality (Glinsukon et al. 1972). The intraperitoneal $L_{50}$ of patulin was $5.9 \mathrm{mg} / \mathrm{kg}$ for rats (Hayes, Phillips and Williams 1978) and 7.5-7.6 mg/kg for mice (Hayes, Phillips and Williams 1978; McKinley and Carlton 1979). The intraperitoneal $L D_{50}$ of cytochalasin $E$ for various adult mice strains ranged from 3.1-3.3 $\mathrm{mg} / \mathrm{kg}$ (Austin, Wind and Brown 1982). The oral $L D_{50}$ for patulin for weanling rats ranged from 108 to $118 \mathrm{mg} / \mathrm{kg}$ (Hayes, Phillips and Williams 1978). However, the oral $L_{50}$ of patulin for mice has varied as determined by different investigators; Hayes and co-workers (1978) established a dose of $17 \mathrm{mg} / \mathrm{kg}$ and McKinley and Carlton (1979) calculated it to be 48 $\mathrm{mg} / \mathrm{kg}$.

Large differences in the concentrations of some of the major $A$. clavatus metabolites were detected from isolates cultured under the same conditions (Table 1). The differences in mycotoxin mixtures and livestock exposure levels might explain the disparity in clinical signs and pathology observed around the world (Gilmour et al. 1989; Sabater-Vilar et al. 2004; Schlosberg et al. 1991). This is corroborated by previous studies in South Africa where patulin, tryptoquivalone and nortryptoquivalone could not be demonstrated in toxic fractions prepared from sorghum beer residue ('maroek') collected during an outbreak (Kellerman et al. 1976). It is interesting to note that Schlosberg and co-workers (1991) compared the syndrome as observed in Israel to those described in South Africa and England and concluded that the South African cultures probably synthesize the widest range of mycotoxins.

The clinical signs of $A$. clavatus poisoning are similar to other tremorgenic syndromes in ruminants caused by indole-diterpenoid mycotoxins. The indole-diterpenoid alkaloids (e.g. paspalinine, paspalitrems, lolitrems) are synthesized by fungi associated with three grass species namely, Paspalum spp., Lolium perenne (perennial ryegrass) and Cynodon dactylon 
(Bermuda grass) (Riet-Correa et al. 2013; Uhlig et al. 2009). The analytical method used included these tremorgenic mycotoxins, but none were detected in any of the $A$. clavatus isolates.

From the analytical results no specific mycotoxin or group of mycotoxins could be implicated as the cause of the disease. All the South African A. clavatus isolates synthesized identical compounds when cultured under similar conditions and there were no non-toxigenic strains. Aspergillus clavatus-infected feed should not be fed to ruminants.

\section{Conflicts of Interest}

None.

\section{Acknowledgements}

This work is based on research supported in part by the National Research Foundation of South Africa (Grant number 103747). The authors would also like to express their gratitude to Marie Smith, Stats4science, who performed the statistical analysis and Danielle Henn who assisted with the drawing of the chemical structures.

\section{References}

Austin WL, Wind M, Brown KS (1982) Differences in the toxicity and teratogenicity of cytochalasin $D$ and $E$ in various mouse strains. Teratology 25:11-18

Botha CJ, Legg MJ, Truter M, Sulyok M (2014) Multitoxin analysis of Aspergillus clavatusinfected feed samples implicated in two outbreaks of neuromycotoxicosis in cattle in South Africa. Onderstepoort J Vet 81(1) doi.org/10.4102/ojvr.v81i1.848 
Büchi G, Luk KC, Kobbe B, Townsend JM (1977) Four new mycotoxins of Aspergillus clavatus related to tryptoquivaline. J Org Chem 42:244-246

Büchi G, Kitaura Y, Yuan SS, Wright HE, Clardy J, Demain AL, Glinsukon T, Hunt N, Wogan GN (1973) Structure of cytochalasin E, a toxic metabolite of Aspergillus clavatus. J Am Chem Soc 95:5423-5425

Clardy J, Springer JP, Koenig T, Büchi G, Matsuo K, Wightman R (1975) Tryptoquivaline and tryptoquivalone, two tremorgenic metabolites of Aspergillus clavatus. J Am Chem Soc 97:663-665

Gilmour JS, Inglis DM, Robb J, Maclean M (1989) A fodder mycotoxicosis of ruminants caused by contamination of a distillery by-product with Aspergillus clavatus. Vet Rec 124:133-135

Glass NL, Donaldson GC (1995) Development of premier sets designed for use with the PCR to amplify conserved genes from filamentous Ascomycetes. Appl Environ Microb 61:1323-1330

Glinsukon T, Yuan SS, Wightman R, Kitaura Y, Büchi G, Shank RC, Wogan GN, Christensen CM (1974) Isolation and purification of cytochalasin E and two tremorgens from Aspergillus clavatus. Plant Foods Man 1:113-119

Hayes AW, Phillips TD, Williams WL (1978) Acute toxicity of patulin. Toxicol Appl Pharm 45:275-276.

Kellerman TS, Coetzer JAW, Naudé TW, Botha CJ (2005) Plant poisonings and mycotoxicoses of livestock in southern Africa, $2^{\text {nd }}$ edn. Oxford University Press, Cape Town 
Kellerman TS, Pienaar JG, Van der Westhuizen GCA, Anderson LAP, Naudé TW (1976) A highly fatal tremorgenic mycotoxicosis of cattle caused by Aspergillus clavatus. Onderstepoort J Vet 43:147-154

Klich MA (2002) Identification of common Aspergillus species. Centraalbureau voor Schimmelcultures, Utrecht

Lopez-Diaz TM, Flannigan B (1997) Production of patulin and cytochalasin E by Aspergillus clavatus during malting of barley and wheat. Int J Food Microbiol 35:129-136

Malachova A, Sulyok M, Beltran E, Berthiller F, Krska R (2014) Optimization and validation of a quantitative liquid chromatography-tandem mass spectrometric method covering 295 bacterial and fungal metabolites including all regulated mycotoxins in four model food matrices. J Chromatogr A 1362:145-156

Malachova A, Sulyok M, Beltran E, Berthiller F, Krska R (2015) Multi-Toxin Determination in Food - The Power of "Dilute and Shoot" Approaches in LC-MS-MS. LC GC Eur 28:542-555

McKenzie RA, Kelly MA, Shivas RG, Gibson JA, Cook PJ, Widderick K, Guilfoyle AF (2004) Aspergillus clavatus tremorgenic neurotoxicosis in cattle fed sprouted grains. Aust Vet J 82:635-638

McKinley ER, Carlton WW (1979) Patulin mycotoxicosis in Swiss ICR mice. Food Cosmet Toxicol 18:181-187

Payne RW (2014) Introduction to GenStat for Windows, $17^{\text {th }}$ edn. VSN International, Hemel Hempstead

Riet-Correa F, Rivero R, Odriozola E, De Lourdes Adrien M, Medeiros RMT, Schild AL (2013) Mycoxicoses of ruminants and horses. J Vet Diagn Invest 25:692-708 
Sabater-Vilar M, Maas RFM, De Bosschere H, Ducatelle R, Fink-Gremmels J (2004) Patulin produced by an Aspergillus clavatus isolated from feed containing malting residues associated with a lethal neurotoxicosis in cattle. Mycopathologia 158:419-426

Samson RA, Visagie CM, Houbraken J, Hong S-B, Hubka V, Klaassen CHW, Perrone G, Seifert KA, Susca A, Tanney JB, Varga J, Kocsubé S, Szigeti G, Yaguchi T, Frisvad JC (2014) Phylogeny, identification and nomenclature of the genus Aspergillus. Stud Mycol 78:141-173

Schlosberg A, Zadikov I, Perl S, Yakobson B, Varod Y, Elad D, Rapoprt E, Handji V (1991) Aspergillus clavatus as the probable cause of a lethal mass neurotoxicosis in sheep. Mycopathologia 114:35-39

Uhlig S, Botha CJ, Vrålstad T, Rolén E, Miles CO (2009) Indole-diterpenes and ergot alkaloids in Cynodon dactylon (Bermuda grass) infected with Claviceps cynodontis from an outbreak of tremors in cattle. J Agric Food Chem 57:11112-11119

Varga J, Due M, Frisvad JC, Samson RA (2007) Taxonomic revision of Aspergillus section Clavati based on molecular, morphological and physiological data. Stud Mycol 59:89106 